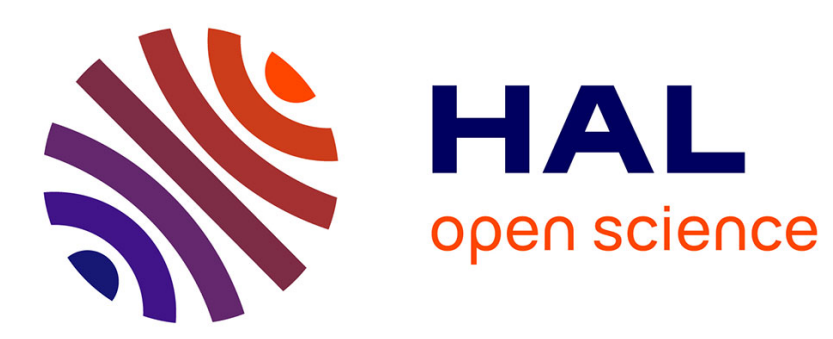

\title{
Introduction. Le capital humain : perspectives historiques et cliométriques
}

\author{
Claude Diebolt, Charlotte Le Chapelain
}

\section{To cite this version:}

Claude Diebolt, Charlotte Le Chapelain. Introduction. Le capital humain: perspectives historiques et cliométriques. Revue d'Economie Politique, 2020, 130 (1), pp.1-4. 10.3917/redp.301.0001 . hal02920362

\section{HAL Id: hal-02920362 \\ https://hal.science/hal-02920362}

Submitted on 25 Aug 2020

HAL is a multi-disciplinary open access archive for the deposit and dissemination of scientific research documents, whether they are published or not. The documents may come from teaching and research institutions in France or abroad, or from public or private research centers.
L'archive ouverte pluridisciplinaire HAL, est destinée au dépôt et à la diffusion de documents scientifiques de niveau recherche, publiés ou non, émanant des établissements d'enseignement et de recherche français ou étrangers, des laboratoires publics ou privés. 


\title{
Introduction : \\ Le capital humain : perspectives historiques et cliométriques
}

\author{
Claude Diebolt*, Charlotte Le Chapelain** \\ *BETA-CNRS, Université de Strasbourg, 61 Avenue de la Forêt noire, 67085 Strasbourg, France. \\ cdiebolt@unistra.fr \\ **CLHDPP, BETA, Université Lyon III, 15 quai Claude Bernard, B.P. 0638, 69239 Lyon Cedex 02, France. \\ charlotte.le-chapelain@univ-lyon3.fr
}

Depuis la «Révolution du capital humain », initiée à la fin des années 1950, l'analyse économique reconnaît le capital humain comme un facteur important de développement et de croissance économique. La contribution du capital humain au processus de croissance demeure toutefois, paradoxalement peut être, sujette à de nombreuses incertitudes. Sa compréhension précise se heurte, presque systématiquement, à la difficulté d'évaluer de manière pertinente les dotations en capital humain. Cette difficulté constitue d'ailleurs la pierre d'achoppement du programme du capital humain depuis son origine. De nos jours, cette difficulté donne lieu à une critique sévère de son programme de recherche, si ce n’est à sa remise en question (voir Hanushek et Woessmann 2015).

Ce numéro de la Revue d'Economie Politique s'est construit face à cette critique et aux enjeux qu'elle suscite. En arrière plan du problème de l'évaluation du capital humain se situent notamment deux faiblesses : une définition nébuleuse du concept et une compréhension très imparfaite des processus à l'origine de la formation du capital humain. L’ambition de ce numéro spécial est de contribuer à éclairer ces enjeux par le recours à une perspective historique. Il réunit des contributions d'histoire économique - qu'elles relèvent de l’histoire de la pensée économique ou de la cliométrie vouées à promouvoir une réflexion enrichie quant au concept de capital humain et aux mécanismes à l'origine de son accumulation.

Venue d'Amérique, la cliométrie, celle de Robert Fogel est, avec son arrivée en Europe, empreinte de malentendus, si ce n’est méprisée pour les intentions délibérément nuisibles qu'on lui prête. Pour la France, dès 1977, Jean Heffer livre ainsi, dans les Annales, avec clairvoyance et sagacité, les raisons de l'inévitable naufrage. Certes, Lévy-Leboyer et Bourguignon (1985), puis Boyer (1989), toujours dans les Annales, en appelleront à de nouvelles alliances... en vain semble-til ! Ce faisant, s'agit-il, sans relâche, de reconsidérer les causes de l'acte manqué et de s'interroger, sans fin, sur les conséquences des opportunités de recherche ratées ? A l'évidence, non et, de grâce, halte aux vestiges du passé ! (Diebolt, 2016, Diebolt et Haupert, 2016ab, 2018, 2019). 
L'emploi de la démarche contrefactuelle comme méthode d'analyse de causalité constitue l'héritage marquant de Fogel. Peut-être est-ce la conséquence du torrent de critique suscité par le désormais illustre Railroads and American Economic Growth (1964) et sa méthode d'analyse qualifiée par certains historiens de «quasi-histoire »? Bien qu'elle fût marquante, l'approche contrefactuelle ne saurait résumer l'apport de Fogel dans l'appréhension des relations causales à l'origine d'un événement historique. En la matière, ce n'est pas tant cette perspective empirique innovante que la nature même de la démarche cliométrique, qu'il a contribué à définir, qui nous apparaît décisive. En fondant la nouvelle histoire économique, Fogel a, en effet, défendu une approche d'analyse assise sur la conjonction de l'analyse historique, d'un ancrage fort dans la théorie économique et de l'emploi des méthodes statistiques et économétriques.

Greasley et Oxley (2010) identifient cinq traditions intellectuelles qui se situent à l'origine de la cliométrie et qui ont façonné son évolution. Ils identifient, tout d'abord, la démarche quantitative, initiée au moins depuis le $\mathrm{XIX}^{\mathrm{e}}$ siècle, avec la construction de séries historiques longues, comme une tradition décisive que prolonge la cliométrie («1. Quantitative History»). Le large mouvement quantitatif qui s’est dessiné dans les années 1960 en sciences sociales, notamment en sciences politiques et en sociologie et qui fut accompagné de l'évolution concomitante de l'informatique, facilitant le traitement de données, constitue une seconde mouvance dans laquelle la cliométrie puise ses fondements («2. Quantitative Social Science and Computing»). Elle a en outre évolué dans les années 1980 en empruntant les apports de l'économétrie et en s’inspirant notamment des analyses d'économétrie appliquée développées dès les années 30 par Tinbergen («3. Econometrics»). L'ancrage dans la théorie économique constitue, dès son origine, la marque de fabrique de la cliométrie. Elle a donné lieu à un renouvellement majeur de l'analyse historique en convoquant les apports théoriques récents et leur évolution («4. Economic Theory»). Enfin, l’évolution de la cliométrie dans les années 1980 s’est nourrie des développements de l’analyse des séries temporelles, sous l’impulsion des travaux d’Engle et Granger notamment («5. Time Series Methods).

À travers ces différentes filiations se dessinent les trois dimensions constitutives de la démarche cliométrique telle que Fogel l'a défendue : l'analyse historique, la mesure et l'ancrage dans la théorie économique- C'est l'alliance de ces trois dimensions dans l'analyse d'un phénomène historique qui, en contribuant notamment à l'identification de représentations erronées de l'histoire économique, a ouvert une perspective nouvelle dans l’identification de liens de causalité en histoire et l'a définitivement différenciée d'une histoire quantitative qui lui préexistait (Margo, 2018, McCloskey 2018, Mongin, 2018).

Champ de prédilection de l’approche cliométrique, l’analyse des déterminants de la croissance s’est rapidement emparée des apports théoriques de la révolution du capital humain, initiée dans les années 1960, sous l’impulsion des travaux de Schultz (1960, 1961), Becker (1964) et Mincer (1958). 
Dans l'examen des relations causales qu'entretiennent le capital humain et la croissance, la synthèse cliométrique s'est d'abord déployée dans deux champs d’investigation privilégiés : le XIX ${ }^{\mathrm{e}}$ siècle et la phase du décollage industriel, puis le $\mathrm{XX}^{\mathrm{e}}$ siècle. C'est en effet à partir de la révolution industrielle et l'accumulation massive de capital humain qui lui sera concomitante que se pose de manière pertinente et complexe la question des liens qui unissent ces deux phénomènes. Au nombre des enjeux liés à l'appréciation pertinente de ce lien, figure tout d'abord l'examen des dotations en capital humain et donc l'évaluation pertinente du concept, par la mesure, dans des contextes historiques distincts. Elle convoque immédiatement la théorie économique, soulignant, en ce domaine, les opacités théoriques du concept de capital humain.

Dans leur contribution, Charlotte Le Chapelain et Sylvère Matéos proposent d'éclairer certaines de ces opacités en examinant la trajectoire intellectuelle d'un des principaux protagonistes de la «Révolution du capital humain», Théodore Schultz. Schultz a été à l'origine d’une réflexion conceptuelle particulièrement riche, qui a contribué de manière déterminante au développement du concept et de la théorie du capital humain. L'objet de cet article consiste à mettre en lumière cette richesse conceptuelle qui demeure relativement sous-estimée. Il éclaire ce faisant, les fondements de la théorie du capital humain mais également ses difficultés, et notamment celle de la compréhension qui demeure très imparfaite, de l'aveu même de Schultz dans les années 1970 - du processus d'accumulation de capital humain.

Ralph Hippe propose une analyse du processus de formation du capital humain en Europe dans une perspective de long terme. Son examen de la distribution régionale du capital humain en Europe durant les deux siècles derniers s'appuie sur une réflexion relative à la pertinence des indicateurs employés pour sa mesure et sur l'analyse des facteurs explicatifs à l’origine des différences de dotation entre les régions. L’auteur souligne que la compréhension de ces hétérogénéités de longterme et des logiques qui, au plan historique, ont présidé aux inégalités de capital humain sont déterminantes quant à la formulation des recommandations des politiques visant à promouvoir l'accumulation de cette forme de capital.

L'impact des inégalités de genre en matière d'accumulation de capital humain est examiné par Faustine Perrin. Sa contribution retrace et analyse les inégalités de capital humain entre hommes et femmes selon le statut marital en France au milieu du XIX ${ }^{\mathrm{e}}$ siècle. Son approche, fondée sur la méthode du Age heaping, met en évidence une influence distincte du statut marital entre hommes et femmes en matière de compétences en écriture et en calcul. L'auteur explore ensuite quatre hypothèses capables d'expliquer ces différences dans le contexte du XIX ${ }^{\mathrm{e}}$ siècle.

Poursuivant une approche comparée, Gabriele Cappelli analyse le processus de convergence régionale, en matière d'éducation primaire, en France et en Italie au XIX ${ }^{\mathrm{e}}$ siècle. Introduisant des estimations nouvelles des ratios bruts de scolarisation primaire ainsi que des dépenses d'éducation au niveau municipal et au niveau de l'Etat, il met en évidence l'existence d'un mouvement de convergence régional antérieur à la mise en œuvre d'une organisation centralisée des systèmes 
éducatifs. Le passage à centralisation est néanmoins identifié comme un élément important d'accélération du processus de convergence régionale, conférant à la politique publique un rôle clé pour le développement de l'éducation dans les deux pays.

Retraçant, au niveau régional, la trajectoire de croissance des Grammar schools en Angleterre entre 1270 et 1700, la contribution d'Alexandra de Pleijt conte deux révolutions éducatives, toutes deux caractérisées par une croissance rapide de l'accumulation de capital humain. La première, entre l'épisode de la Peste Noire et les années 1530, est associée au régime démographique consécutif à l'épisode de peste noire, identifié comme avoir créé les conditions favorables à l'accumulation de capital humain. La seconde révolution, durant la seconde moitié du dix-septième siècle, fut concentrée dans les zones d'urbanisation rapide qui ont constitué un peu plus tard les grands centres industriels anglais. L'analyse suggère l'émergence, durant la phase pré-industrielle, d'une demande de travail qualifié émanant du secteur des services, dont l'essor a précédé celui du secteur manufacturier, caractéristique quant à lui de la phase de la Révolution industrielle.

\section{Références}

Becker, G.S. (1964), Human Capital. A Theoretical and Empirical Analysis, with Special Reference to Education, New York: Columbia University Press.

Boyer, R. (1989) Économie et histoire : vers de nouvelles alliances. Annales. Économies, Sociétés, Civilisations, 6, 1397-1426.

Diebolt, C. (2016) Cliometrica after 10 years: Definition and Principles of Cliometric Research, Cliometrica, 10 (1), 1-4.

Diebolt, C., Haupert, M. Eds. (2016a), Handbook of Cliometrics. Berlin: Springer.

Diebolt, C., Haupert M. (2016b) Clio's Contributions to Economics and History, Revue d'Economie Politique, 126 (5), 971-989.

Diebolt, C., Haupert M. (2018) A Cliometric Counterfactual: What If There Had Been Neither Fogel nor North?, Cliometrica, 12 (3), 407-434.

Diebolt, C., Haupert, M. Eds. (2019), Handbook of Cliometrics. $2^{\text {nd }}$ Edition, Berlin: Springer.

Fogel, R. W. (1964). Railroads and American Economic Growth: Essays in Econometric History. Baltimore: Johns Hopkins Press.

Greasley D. et Oxley L. (2010), Clio and the Economist: Making Historian Count, Journal of Economic Surveys, 24 (5), 755-774.

Hanushek, E., Woessmann, L. (2015). The Knowledge Capital of Nations. Education and the Economics of Growth. Cambridge, MA: MIT Press.

Heffer, J. (1977), Une histoire scientifique : la nouvelle histoire économique, Annales. Économies, Sociétés, Civilisations, 4, 1977, pp. 824-842. 
Lévy-Leboyer, M., Bourguignon, F. (1985). L'économie française au XIXe siècle : analyse macroéconomique. Paris : Economica.

Margo, B. (2018), The Integration of Economic History into Economics, Cliometrica, 12 (3), 377-406.

McCloskey, D. (2018), Getting Over Naïve Scientism c. 1950: What Fogel and North Got Wrong, Cliometrica, 12 (3), 435-449.

Mincer, J. (1958), Investment in Human Capital and Personal Income Distribution, Journal of Political Economy, 66 (4), 281-302.

Schultz, T. W. (1960), Capital Formation by Education, Journal of Political Economy, 68 (6), 571583.

Schultz, T. W. (1961), Investment in Human Capital, American Economic Review, 51 (1), 1-17. 